\section{Commentary: The importance of being predictable: Postoperative atrial fibrillation}

\author{
Arnaldo Dimagli, MD, Ettorino Di Tommaso, MD, \\ and Vito Domenico Bruno, MD, PhD
}

Postoperative atrial fibrillation (pAF) represents a common event complicating the postoperative course of $20 \%$ to $40 \%$ of patients undergoing cardiac surgery. ${ }^{1}$ Although considered for a long time a benign and transient event, recent evidence has highlighted an association with increased mortality and morbidity, in particular cerebrovascular accidents. ${ }^{2}$ One of the main triggering factors for $\mathrm{pAF}$ is the inflammatory status induced by the surgical trauma (eg, cardiopulmonary bypass). ${ }^{1}$

In the current issue of the Journal, Liu and colleagues ${ }^{3}$ investigated the role of leucocyte-derived myeloperoxidase (MPO), an important mediator of cardiac surgery-related inflammation, as a predictor of $\mathrm{pAF}$ by measuring its preoperative and postoperative concentrations in the blood and pericardial fluid of patients undergoing coronary artery bypass grafting surgery. Notably, authors also tried to justify the pivotal role of MPO in canine models by studying the MPO-induced structural and electrical remodeling of atria.

The authors must be congratulated for this impactful piece of evidence regarding such a common complication and for highlighting the importance of including inflammatory markers in the prediction tools for pAF. The model presented in the paper showed that when MPO levels were introduced in the prediction rule, together with clinical and surgical parameters, the predictive accuracy of the model was outstanding (area under the curve 0.90) and improved compared with the model built on clinical and surgical variables only. This finding paves the way for the identification of new optimal predictive biomarkers and

\footnotetext{
From the Bristol Heart Institute, University of Bristol, Bristol, United Kingdom. Disclosures: The authors reported no conflicts of interest.

The Journal policy requires editors and reviewers to disclose conflicts of interest and to decline handling or reviewing manuscripts for which they may have a conflict of interest. The editors and reviewers of this article have no conflicts of interest.

Received for publication June 29, 2021; revisions received June 29, 2021; accepted for publication June 30, 2021; available ahead of print July 6, 2021

Address for reprints: Arnaldo Dimagli, MD, Bristol Royal Infirmary, Office Room 84,

Level 7, Upper Maudlin St, BS28HW Bristol, United Kingdom (E-mail: arnaldo.

dimagli@bristol.ac.uk).

J Thorac Cardiovasc Surg 2023;165:1078-9

$0022-5223 / \$ 36.00$

Copyright (c) 2021 by The American Association for Thoracic Surgery

https://doi.org/10.1016/j.jtcvs.2021.06.059
}



Arnaldo Dimagli, MD, Ettorino Di Tommaso, MD, and Vito Domenico Bruno, MD, PhD

\section{CENTRAL MESSAGE \\ The benefits of a properly con- ducted regression model are very helpful in creating predictive clinical instruments.}

their inclusion in clinical prediction models. In this setting, the statistical methods used have a pivotal role and should be carefully planned and conducted. When building a predictive model, it is of utmost relevance to create a useful and practical tool. For instance, the variables included in the model should be easily and readily measured as to have the widest possible applicability in the daily clinical practice. In the study by Liu and colleagues, some concerns could be raised from the inclusion of the serum MPO levels measured at 6 hours postoperatively because this variable can be obtained only few hours after surgery. However, pAF usually occurs between 24 hours and 48 hours after surgery ${ }^{4}$ and therefore measurement of MPO at 6 hours should not invalidate the general applicability of the model.

Moreover, predictive model development involves the crucial step of model validation that allows to assess model performance in a different setting from the one in which the model was developed. Unfortunately, the prediction rule proposed by Liu and colleagues was not validated by any means. Validation can be performed using the same data by bootstrapping or $k$-fold cross-validation, or by splitting the sample in a training and a validation set, or by using a different dataset ("external validation"). It will be essential that the model is validated and maybe also retrained in a bigger data sample. As such, it is not possible to speculate on the actual prediction performance of this tool and investigate the potential presence of overfitting.

Also, Liu and colleagues presented a quite small sample size for the development of a prediction rule, and there was a very high proportion of patients undergoing off-pump grafting. This limits the conclusions that can be drawn 
regarding the performance and generalizability of the models.

Concluding, Liu and colleagues must be congratulated for their work, which highlights the importance of integrating inflammatory parameters in the prediction rules for pAF, which is very much needed. The necessity of a prediction tool is dictated by the need to correctly identify patients at high risk of developing pAF. Once identified, these patients can benefit from prophylactic treatments (eg, amiodarone, beta-blockers) and the overall health care expenditure associated with the development of pAF could be reduced. $^{5}$

\section{References}

1. Gudbjartsson T, Helgadottir S, Sigurdsson MI, Taha A, Jeppsson A Christensen TD, et al. New-onset postoperative atrial fibrillation after heart surgery. Acta Anaesthesiol Scand. 2020;64:145-55.

2. Benedetto U, Gaudino MF, Dimagli A, Gerry S, Gray A, Lees B, et al. Postoperative atrial fibrillation and long-term risk of stroke after isolated coronary artery bypass graft surgery. Circulation. 2020;142:1320-9.

3. Liu Y, Yu M, Wu Y, Wu F, Feng X, Zhao H. Myeloperoxidase in the pericardia fluid improves the performance of prediction rules for postoperative atrial fibrillation. J Thorac Cardiovasc Surg. 2023;165:1064-77.e8.

4. Mathew JP, Fontes ML, Tudor IC, Ramsay J, Duke P, Mazer CD, et al. A multicenter risk index for atrial fibrillation after cardiac surgery. J Am Med Assoc. 2004;291:1720-9.

5. Hernández-Leiva E, Alvarado P, Dennis RJ. Postoperative atrial fibrillation: evaluation of its economic impact on the costs of cardiac surgery. Braz J Cardiovasc Surg. 2019;34:179-86. 